Editorial

\title{
Acknowledgment to Reviewers of Diagnostics in 2020
}

\section{Diagnostics Editorial Office}

MDPI AG, St. Alban-Anlage 66, 4052 Basel, Switzerland

Peer review is the driving force of journal development, and reviewers are gatekeepers who ensure that Diagnostics maintains its standards for the high quality of its published papers. Thanks to the cooperation of our reviewers, in 2020, the median time to first decision was 16 days and the median time to publication was 33 days. The editors would like to express their sincere gratitude to the following reviewers for their precious time and dedication, regardless of whether the papers were finally published:

Aarntzen, Erik

Abdelbasset, Walid Kamal

Abdul-Rahman, Omar A.

Abe, Tomoyuki

Abomaray, Fawaz Mohamed

Acampa, Maurizio

Acharjee, Animesh

Achiron, Asaf

Acién, Pedro

Acker, Jason P.

Acri, Giuseppe

Adam, Margaret P.

Adamczewski, Zbigniew

Adamiok-Ostrowska, Anna

Adamopoulos, Panagiotis

Afat, Saif

Afrantou, Theodora

Agarwal, Sumit

Aggeli, Constantina

Agresta, Ferdinando

Ahmad, Khurshid

Ahn, Yong

Ai, Yong

Aich, Satyabrata

Aizenberg, Michele

Akagi, Satoshi

Alaimo, Alessandro

Al-antari, Mugahed A.

Albano, Domenico

Alberti, Daniele

Albertolle, Matthew E.

Albu, Silviu

Alcalde, Mireia

Alcántara-Ortigoza, Miguel Angel

Alexander, Adrian
Ali, Lamiaa M. A.

Ali, Zulfiqur

Al-Imam, Ahmed

Alizargar, Javad

Aljabali, Alaa A. A.

Alkan, Michael

Alkatout, Ibrahim

Alkhateeb, Abedalrhman

Allen, Richard C.

Al-Maawi, Sarah

Almansa, Inmaculada

Almeida, Luis

Aloisi, Anna Maria

Alongi, Pierpaolo

Alpar, Donat

Alpar, Orcan

Altamura, Claudia

Altini, Corinna

Altintas, Mehmet M.

Altmann, Friedrich

Álvaro, Tomás

Alzubaidi, Laith

Amadio, Peter

Amado, Sandra

Amaricai, Elena

Amedei, Amedeo

Amin, Nivee P.

Amit, Moran

Amorim, João

Amornyotin, Somchai

Amzar, Daniela

Ana Maria Cristina, Tancu

Anagnostis, Athanasios

Anania, Maria Chiara

Ananthakrishnan, Soundaram Jeevarathinam 
Andaloro, Claudio

Andica, Christina

Andrade De Jesus, Danilo

Andrearczyk, Vincent

Andreetti, Claudio

Andrius, Kazlauskas

Andrukhov, Oleh

Andrzej, Nowicki

Anemona, Lucia

Ang, Eng Tat

Angelini, Corrado

Angelousi, Anna

Angheben, Andrea

Anghel, Larisa

Anitescu, Cosmin

Annaswamy, Thiru M.

Ansani, Lucia

Antignani, Pier Luigi

Antigua, Khristine Joy C.

Antonakakis, Marios

Antonela Antoniu, Sabina

Antoniu, Sabina Antonela

Antony, Benny S. Eathakkattu

Antony, Reuben

Anwar, Syed Muhammad

Aoki, Mitsuhiro

Aoki, Takatoshi

Aoude, Lauren

Applegate, Tanya

Apra, Caroline

Aran, Veronica

Arandjelović, Ognjen

Arason, Adalgeir

Ardeleanu, Valeriu

Arena, Alessandro

Argula, Rahul Gupta

Arizono, Shigeki

Armellini, Elia

Armengot-Carceller, Miguel

Arnedo, Maria

Arosio, Paolo

Artigas, Carlos

Arul, Michael

Ashida, Reiko

Asimaki, Angeliki

Asmundo, Alessio

Aso, Yoshimasa

Assi, Mohamad

Assinder, Stephen

Ata-Ali, Javier

Auriti, Cinzia
Aushev, Vasily

Avanzo, Michele

Ávila, Julio

Ayaki, Masahiko

Ayat, Nadia

Aydede, Sema K.

Ayoub, Ashraf

Azizian, Azadeh

Babar, Ghufran

Bacalbaşa, Nicolae

Baffy, Gyorgy

Bagci, Ulas

Bagiu, Iulia-Cristina

Bahmad, Hisham

Baig, Imran

Bailey, Simon

Bainbridge, Jacci

Bajan, Sarah

Bajwa, Amandeep

Bakhshinejad, Ali

Bakht, Martin

Balaban, Daniel Vasile

Baldacci, Filippo

Baloyannis, Stavros J.

Baltzer, Pascal A. T.

Bamatraf, Saeed M.

Bandres-Ciga, Sara

Baniahmad, Aria

Bansal, Aditya

Bär, Séverine

Baratella, Elisa

Barauskas, Giedrius

Barbee, Lindley A.

Barberis, Massimo

Barbosa, Ana Isabel

Barbosa, Flávia Leão

Barca, Amilcare

Barda, Noam

Barnes, Anna

Barnett, Brad

Barra, Fabio

Barran, Perdita E.

Bar-Shalom, Rachel

Bartel, Sabine

Bartnik, Ewa

Bartoli, Francesco

Barton, Matthew J.

Bartulos, Oscar

Barua, Animesh

Basak, Sujit

Bassareo, Pier Paolo 
Batchuluun, Ganbayar

Battistelli, Cecilia

Bauckneht, Matteo

Baumrucker, Steven

Baxter, Eva

Bay, Peter

Beasley, Shannon

Bechsgaard, Thor

Bechter, Karl

Bednarz-Knoll, Natalia

Beer, Ambros J.

Behringer, Erik

Bel'skaya, Lyudmila

Belcher, John R.

Bellin, Marie France

Belluzzi, Elisa

Bender, Steven D.

Benko, Zsofia

Bennardo, Francesco

Bennardo, Luigi

Beňuš, Radoslav

Benyhe, Sandor

Benzaquen, Michael

Berardo, Clarissa

Beresford, David

Berezin, Alexander

Bergandi, Loredana

Bergougnoux, Anne

Bernabéu, Carmelo

Berretti, Stefano

Bertelli, Elena

Berthelot, Laureline

Bertoli, Gloria

Bertomeu-González, Vicente

Bertucci, Emma

Bevc, Sebastjan

Bhat, Ambarish P.

Bhatia, Vikram

Bhute, Shrikant

Białynicki-Birula, Rafał

Bianconi, Francesco

Bikov, Andras

Binda, Cecilia

Biswas, Arijit

Biswas, Santanu

Bis-Wencel, Hanna

Blanc, Dominique

Blank, Andreas Christian

Blanton, Robert M.

Blasi, Elisabetta

Błaszczyszyn, Monika
Blaumeiser, Bettina

Bleilevens, Christian

Bloch, Karin Markenroth

Boaventura, Paula

Boccolini, Daniela

Bödör, Csaba

Böer, Klas

Bohannon, Richard W.

Bolboacă, Sorana D.

Boldorini, Renzo

Bologna, Ronell

Bonda, Tomasz

Bonetto, Andrea

Bongiorno, Dafne

Bonham, Jim

Bonifazi, Martina

Bordonaro, Michael

Borghesi, Andrea

Borgia, Jeffrey A.

Borisova, Ekaterina

Boros, Laszlo

Borovac, Josip A.

Borroni, Davide

Bosch, Xavier

Bose, Tanima

Botella, Luisa María

Botnar, Rene

Botti, Gerardo

Botton, Thomas

Boubchir, Larbi

Bouhrara, Mustapha

Bowling, Heather L.

Boysen, Reinhard

Bozso, Sabin J.

Bracht, Thilo

Brady, Adrian

Brady, Zoe

Bragazzi, Nicola Luigi

Braicu, Cornelia

Brak, Ivan

Bratashov, Daniil N.

Brcic, Luka

Brcic, Mario

Bressem, Keno-Kyrill

Breuker, Anja

Brieger, Angela

Brigante, Giulia

Broderick, Patricia A.

Broggi, Morgan

Brogi, Simone

Brogna, Claudia 
Bromberg, Mark B.

Brosens, Erwin

Brown, Christopher Lyon

Broza, Yoav

Brozek-Pluska, Beata

Brun, Emmanuel

Bruneel, Arnaud

Brunetti, Oronzo

Bruno, Antonio Giulio

Brustmann, Hermann

Bruzzese, Dario

Bryere, Joséphine

Buckley, Liz

Bucolo, Claudio

Budzevich, Mikalai M.

Budzik, Jean-François

Bueno-Campaña, Mercedes

Bueno-Crespo, Andrés

Buffi, Nicolò Maria

Bujan, Julia

Büki, András

Bultot, Laurent

Bundschuh, Ralph A.

Bungau, Simona Gabriela

Buonaguro, Elisabetta Filomena

Burattini, Laura

Burger, Michael C.

Butkauskas, Dalius

Buza, Krisztian

Byeon, Haewon

Byoung-Hee, Lee

Caggiati, Alberto

Caiati, Carlo

Caivano, Domenico

Calcagni, Giulio

Caldara, Marina

Caldarella, Carmelo

Caliò, Anna

Callegaro, Annapaola

Caltabiano, Rosario

Calvano, Cosima Damiana

Calvieri, Stefano

Calvo Lobo, César

Camara Serrano, Juan

Cameselle-Teijeiro, Josè Manuel

Campagna, Giuseppe

Campana, Luca Giovanni

Campus, Guglielmo

Candela, Diaz-Canestro

Cândido Carvalho, Kátia

Cannella, Roberto
Canti, Valentina

Canty, David J.

Cao, Jian

Caparros-Gonzalez, Rafael A.

Capoccia, Massimo

Caponio, Vito Carlo Alberto

Caporali, Leonardo

Cappelletti, Vera

Captur, Gabriella

Capurso, Gabriele

Carbone, Antonino

Carbone, Michele

Carboni, Nicola

Carinelli, Silvestro

Carney, A Simon

Caroli, Anna

Carpenter, Lewis

Carpi, Sara

Carraro, Ugo

Carrier, Paul

Carriero, Alessandro

Carrilho, Eunice

Carrizzo, Albino

Carugno, Jose

Caruntu, Constantin

Caruso, Damiano

Carvalho, Beatriz

Casals, Gregori

Casari, Alice

Casas, François

Casas, Rosa M.

Cascini, Giuseppe Lucio

Casella, Michela

Caserta, Donatella

Casiraghi, Elena

Castresana, Javier S.

Castro, Andre

Castro, Pedro

Catalano, Antonino

Catanzaro, John N.

Caterino, Umberto

Cavalli, Loredana

Caviglia, Gian Paolo

Cavoretto, Paolo

Cazzolla, Angela Pia

Cebova, Martina

Cécile, Delettre

Celi, Alessandro

Cellina, Michaela

Ceppi, Lorenzo

Cerrolaza, Juan J. 
Cervantes, Jorge

Cervigón, Raquel

Chace, Donald H.

Chadwick, Philip

Chadwick, Raymond J.

Chan, Kannie

Chan, Kwok-Hung

Chang, Chia-Chen

Chang, Hyeyoon

Chang, Jia-Feng

Chang, Ke-Vin

Chang, Shin-Tsu

Chang, Yao-Lung

Chao, Chia-Ter

Chao, Chien-Ming

Chao, Day-Yu

Charabidze, Damien

Chaudhary, Pankaj

Chauhan, Neeraj

Chaveeva, Petya

Chavez-Santoscoy, Rocio Alejandra

Chen, Argon

Chen, Chien-Chin

Chen, Chun-Jung

Chen, Hsiang-Yin

Chen, Huarong

Chen, Jyh-Cheng

Chen, Ke-Cheng

Chen, Kevin T.

Chen, Kuan-Fu

Chen, Kuan-Hsing

Chen, Liben

Chen, Mingsheng

Chen, Shing-Jye

Chen, Tai-Heng

Chen, Xiaoyan

Chen, Yann-Jang

Chen, Yei-Tsung

Chen, Yu-Chun

Chen, Zhitong

Cheng, Chun-An

Cheng, Juei-Tang

Chepelev, Leonid

Cheungpasitporn, Wisit

Chi, Gerald

Chi, Yung-Wei

Chiaramello, Anne

Chiaramonte, Rita

Chicklore, Sugama

Chien, Yin-Hsiu

Chigbu, De Gaulle I.
Chinegwundoh, Francis

Ching, Congo Tak Shing

Chintalapudi, Sumana

Chioncel, Ovidiu

Chitale, Shalaka

Chizzolini, Carlo

Chlanda, Petr

Cho, Jaeho

Cho, Kang Su

Choi, Hojong

Choi, Joon Hyuk

Choi, Woo June

Chong, Cher-Rin

Chong, Yosep

Choo, Pei Ling

Choudhary, Sanjeev

Chowdhury, Imran Hussain

Chrcanovic, Bruno

Christopoulos, Petros

Chroni, Elisabeth

Chu, Chun Hung

Chuah, Seng-Kee

Chuang, Wen-Yu

Chulanov, Vladmir

Chun, Dong-il

Chung, Chi-Li

Chung, Yong-An

Cianci, Pasquale

Ciancio, Gaetano

Cianciolo, Giuseppe

Cicone, Francesco

Ciebiera, Michal

Cilia, Giovanni

Cillero-Pastor, Berta

Ciobica, Alin

Cipakova, Ingrid

Cipolloni, Luigi

Civit, Anton

Clarke, Luka Alexander

Clemens, Robert K.

Clement, Yuen

Coccheri, Sergio

Cocco, Stefania

Cofta, Szczepan

Cohen, Michael B.

Coletta, Ricardo

Colicchia, Michele

Collado, Aida

Collier, Andrew

Collini, Federica

Colomo, Lluís 
Colon Saez, Jose

Colosi, Horațiu Alexandru

Colosimo, Carlo

Conceicao, Raquel

Conti, Pio

Conti, Valeria

Contreras-Aguilar, María Dolores

Coppel, Yannick

Coraci, Daniele

Corda, Andrea

Cornejo, Alberto

Cornelis, François

Cornelius, Denise

Corrigan, Damion K.

Corris, Paul A.

Cosby, Louise

Cosmin Mihai, Vesa

Costa, Joana

Costa, Pedro

Cottereau, Anne-Ségolène

Coucke, Wim

Cousins, Joseph

Coustau, Christine

Coutinho, Maria Francisca

Cowan, Kyra J.

Cowden Dahl, Karen

Cozzani, Emanuele

Crawford, Sean

Creytens, David

Crino, Stefano Francesco

Cristache, Corina Marilena

Crnkovic, Ana

Croce', Lory Saveria

Cronin-Golomb, Mark

Crupi, Vincenza

Cubiella, Joaquin

Cui, Yanxiang

Cuocolo, Renato

Curic, Goran

Cymbaluk, Aneta

Czakó, László

Czekierdowski, Artur

Czimmer, József

Czuczwar, Mirosław

D'Agostino, Donato

D'Aguanno, Simona

D'Argenio, Valeria

D'Arrigo, Sonia

D'Elios, Mario Milco

D'Orazi, Valerio

D'Orsi, Beatrice
Dąbrowska-Kugacka, Alicja

Dailey, George E.

Daimi, Houria

Dal Col, Jessica

Dalessandri, Domenico

Dandekar, Aditya

Danilowicz-Szymanowicz, Ludmila

Darocha, Szymon Darocha

Das, Bibhuti Bhusan

Dasí, Francisco

David, Vlad Laurentiu

Davis, Mellar P.

Dawra, Rajinder

De Backer, Wilfried

De Baets, Liesbet

De Barrios, Oriol

De Berardis, Domenico

De Cobelli, Ottavio

De Falco, Valentina

De Franciscis, Pasquale

De Geus-Oei, Lioe-Fee

De Jonge, Hugo

De La Luz Garcia-Hernandez, Maria

De Leo, Antonio

De Mendoza, Carmen

De Miguel, Diego

De Montpréville, Vincent

De Oliveira, Mario Anderson

De Pauw, Joke

De Reijke, T. M.

De Reijke, Theo M.

De Ruiter, Godard C. W.

De Sanctis, Juan Bautista

De Visser, Marianne

De Zinis, Luca Oscar Redaelli

Dean, Frank

Deán-Ben, Xosé Luís

Deandreis, Désirée

De-Bandt, Jean-Pascal

Dedhia, Priya

Deftereos, Georgios

Deguelte, Sophie

Del Barrio, Melisa

Delacour, Hervé

De-la-Cruz-Torres, Blanca

De-los-Santos-Álvarez, Noemí

Delvaux, Nicolas

Delvecchio, Maurizio

Demyanets, Svitlana

Depledge, Daniel

Derlin, Thorsten 
Deshpande, Gauravi

DeStefano, Christin B.

Destrempes, François

Dhama, Kuldeep

Di Agostino, Silvia

Di Bella, Gianluca

Di Diego, Jose

Di Domenico, Giovanni

Di Donato, Marzia

Di Gennaro, Francesco

Di Girolamo, Stefano

Di Lorenzo, Francesco

Di Luciano, Carmela

Di Profio, Federica

Di Spiezio Sardo, Attilio

Di Stasio, Enrico

Di Tommaso, Luigi

Diamantopoulos, Andreas

Dias, Francisca

Didona, Dario

Dieks, Jana-Katharina

Dietrich, Charles

Dinets, Andrii V.

Dionne, Audrey

Diorio, Caroline

Dir, Jan

Dittmann, Helmut

Divjak, Eugen

Dłuski, Dominik

Dmochowski, Marian

Do, Minh Truong

Docea, Anca Oana

Docimo, Ludovico

Doddapattar, Prakash

Domanin, Maurizio

Domenici, Giacomo

Donati, Marcello

Dong, Qinglin

Donnelly, Callum George

Dorman, David

Dosoky, Noura

Dou, Laetitia

Doulberis, Michael

Dresselaers, Tom

Driessen, Gertjan J.

Drummond, Gordon B.

Druszczynska, Magdalena

$\mathrm{Du}, \mathrm{Ke}$

Dubrova, Yuri

Ducarme, Guillaume

Duchene, Bernard
Dunkley, Benjamin T.

Dunowska, Magdalena

Duong, Phu

Duong-Quy, Sy

Duraturo, Francesca

Durazzo, Alessandra

Dutta, Aniruddha

Duzgunes, Nejat

Dzięgiel, Piotr

Dziurkowska, Ewelina

Eboigbodin, Kevin

Ecke, Thorsten Holger

Edelman, Bradley

Edlefsen, Paul T.

Eeckhoute, Jérôme

Egenlauf, Benjamin

Ehrenpreis, Eli D.

Eid, Nabil

Eiring, Anna

Ekpo, Ernest U.

El Hadi, Hamza

El Midaoui, Adil

Elkins, Kelly

Ellmann, Stephan

Elsayad, Khaled

El-Sheikh Ali, Hossam

Endo, Makoto

Enninga, Elizabeth Ann L.

Erdmann, Kati

Ereminienè, Eglè

Eriksson Steigen, Sonja

Erovic, Boban M.

Escudero-Pérez, Beatriz

Esfahani, Siavash

Espa, Stefania

Esworthy, Robert Steven

Eun, Jung Woo

Evangeliou, Athanasios

Evans, Catherine J.

Evans, Dafydd Gareth

Exbrayat, Jean-Marie

Exindari, Maria

Eykyn, Thomas

Ezhov, Marat

Fabien, Forest

Facciorusso, Antonio

Fadda, Gianluca

Fadda, Guido

Faggioni, Lorenzo

Fagioli, Franca

Falzone, Luca 
Fan, Hueng-Chuen

Fanizzi, Annarita

Fanti, Alessandro

Farina, Nicholas

Faron, Anton

Farrell, Philip M.

Farschtschi, Said Chosro

Fatima, Iram

Faustino, Ana

Fedele, Francesco

Federico, Raveglia

Fehr, André

Feier, Diana Sorina

Feier, Horea

Feng, Huicheng

Feng, Wanjuan

Feodorova, Valentina A.

Feregrino-Pérez, Ana A.

Fernandes Fagundes, Nathalia

Fernández De Las Peñas, César

Fernandez, Patricia

Fernández-Lázaro, Diego

Fernandez-Matias, Ruben

Fernando, Wyss

Ferrara, Francesco

Ferrara, Mariantonia

Ferrara, Pietro

Ferrarazzo, Giulia

Ferrari, Cristina

Ferrari, Federico

Ferreira, Fernando

Ferrero, Simone

Ferri, Flaminia

Ferro, Matteo

Filho, Julio Ricarte

Filip, Stanislav S.

Filippiadis, Dimitrios K.

Filippiadis, Dimitris

Finelli, Carmine

Fineschi, Vittorio

Fiorillo, Luca

Fisse, Anna Lena

Fiz, Francesco

Flores, Idhaliz

Flores-Guerrero, Jose L.

Floria, Mariana

Fochi, Stefania

Foecking, Melanie

Foguel, Marcos Vinicius

Font, Albert

Forest, Fabien
Forget, Patrice

Forlano, Roberta

Forster, Jameson

Forte, Maurizio

Fosbøl, Marie Øbro

Foster, Kenneth R.

Foti, Daniela P.

Fourati, Slim

Frame, Leigh A.

Francesco, Nudi

Francis, Ian C.

Franza, Laura

Frati, Paola

Frauenfelder, Thomas

Fredriksson, Ingemar

Friedmacher, Florian

Frietze, Seth

Frigy, Attila

Fritz, Jan

Froelich, Matthias F.

Frulloni, Luca

Fu, Pin-Kuei

Fujioka, Tomoyuki

Fujishima, Hiroshi

Fujita, Toshitsugu

Fukuda, Akira

Fukui, Atsushi

Fukumoto, Yoshihiro

Fukumura, Yuki

Fukushima, Hiroshi

Gabel, Kelsey

Gabelloni, Michela

Gach, Agnieszka

Gadda, Giacomo

Gaddi, Antonio Vittorino

Gaffney, Christopher

Gagliardo, Cesare

Gagniuc, Paul

Gajewska, Danuta

Galan Mercant, Alejandro

Galani, Vasiliki A.

Galán-Sánchez, Fátima

Galati, Francesca

Galinetto, Pietro

Gall Troselj, Koraljka

Gallagher, Philip M.

Gallego-Colon, Enrique J.

Galvosas, Petrik

Gama, Adelina

Gambini, Juan

Ganau, Mario 
Gancarz, Marek

Gandham, Anoohya

Gao, Xiaoyi Raymond

Garbers, Christoph

García, Montserrat

García-Vicente, Ana María

Garg, Bhavuk

Garinis, George

Gascho, Dominic

Gąsior-Perczak, Danuta

Gaspar, Phyllis

Gasperini, Serena

Gaur, Nishtha

Gaviria, Manuel

Gawel, Damian

$\mathrm{Ge}$, Xiaodong

Geddes-McAlister, Jennifer

Geisler, Ramsia

Gelati, Matteo

Gelb, Michael

Gelsomino, Sandro

Gentili, Francesco

Gentilin, Erica

Georgiev, Georgi As

Gerke, Oke

Germana, Antonino

Gervasi, Rita

Ghanbarzadeh-Dagheyan, Ashkan

Ghandour, Rashed

Gherman, Bogdan

Ghidini, Michele

Ghio, Stefano

Ghiroldi, Andrea

Giammarile, Francesco

Giampieri, Riccardo

Gianfredi, Vincenza

Giangregorio, Francesco

Giannakopoulos, Panteleimon

Giannella, Luca

Giannone, Gaia

Giannotti, Elisabetta

Gianturco, Luigi

Gibbs, Peter

Gibson, James B.

Giebultowicz, Joanna

Gilmour, Susan M.

Gioia, Sara

Giordano, Guido

Giordano, Raffaele

Giordano, Salvatore

Giotakis, Aris I.
Gioulekas, Fotios

Giovagnoli, Raffaela

Giovannelli, Pia

Giraudo, Chiara

Giri, Hemant

Gironella, Meritxell

Gish, Robert

Giudice, Giuseppe Lo

Giuffrè, Mauro

Giungato, Pasquale

Gladysheva, Inna P.

Glaudemans, Andor

Glowacz, Adam

Glushakov, Alexander

Gnanasundram, Sivakumar Vadivel

Godde, Kanya

Goel, Pratibha

Goergen, Craig J.

Goldhawk, Donna E.

Goldis, Adrian

Gołębiowski, Tomasz

Golubovskaya, Vita

Gómez-Barrero, Susana

Gonzalez, Gabriel

Gonzalez, German

Gonzalez, Rogelio

Gonzalez-Penas, Javier

Gorczynski, Adam

Gordeeva, Olga

Gori, Tommaso

Gormsen, Lars C.

Gorrell, Mark

Goto, Katsumasa

Goto, Taichiro

Govindarajan, Sindhuja Tirumalai

Goździewska-Harłajczuk, Karolina

Grabarek, Beniamin Oskar

Grabmüller, Melanie

Grady, Erin E.

Graham, Sheila

Grant, George

Grasso, Giuseppe

Gray, Michael D.

Graziani, Maristella

Greim, Helmut

Greuter, Marcel

Grimm, Alexander

Grimm, Marcus O. W.

Gris, Denis

Grizzi, Fabio

Grochowski, Cezary 
Groeneveld, Dafna

Grošelj, Urh

Grosse, Scott D.

Grymowicz, Monika

Grzanka, Dariusz

$\mathrm{Gu}$, Bin

Guadagni, Stefano

Guagnano, Maria Teresa

Guarnieri, Gabriella

Guembe, Maria

Guenancia, Charles

Guerriero, Stefano

Guglielmi, Valeria

Guglielmo, Priscilla

Guillaud, Martial

Gulei, Diana

Gulino, Ferdinando Antonio

Guo, Yanhui

Gupta, Kuldeep

Gupta, Mohit

Gupta, Vijayalaxmi

Gursoy, Mervi

Gutte, Henrik

Gyselaers, Wilfried

Habanova, Marta

Habiba, Marwan

Hafer, Carsten

Haider, Noman

Haijes, Hanneke

Haj-Mirzaian, Arya

Hall, Kate

Hallac, Rami

Halloran, Kieran

Halmosi, Róbert

Halsall, David J.

Ham, Won-sik

Hamaguchi, Shogo

Hamedpour, Vahid

Hamm, Robert

Han, Der-Sheng

Hanai, Jun-ichi

Hanas, Jay

Hansen, Søren Baarsgaard

Hanssens, Laurence

Hapuarachchi, Hapuarachchige Chanditha

Hardwick, James P.

Hargreaves, Iain

Harris-Love, Michael O.

Hartmann, Jan

Hassaballah, M.

Hatzl, Stefan
Havre, Roald Flesland

Hawkins, Federico

Heaney, J. L.

Hedfalk, Kristina

Hedman, Klaus

Hedriana, Herman Locsin

Heise, Bettina

Heitmar, Rebekka

Helm, Matthew F.

Heng, Xiao

Hénique, Carole

Hennerici, Michael G.

Henrique, Rui

Hepojoki, Satu

Hernandez, Frank

Hernández, Susana

Hernandez-Vaquero, Daniel

Hernes, Marcin

Hervás Pérez, Juan Pablo

Hervouet, Eric

Herynek, Vít

Hida, Richard

Hikichi, Takuto

Hildebrandt, Malene Grubbe

Hildreth, Blake

Hilhorst, Marc

Hingorani, Dina

Hingorani, Rittu

Hippensteel, Joseph

Hirai, Keita

Hirano, Tsunahiko

Hirata, Kenji

Hlaváč, Viktor

Ho, Chichun

Ho, Shih-Ching

Hobolth, Lise

Hoeschen, Christoph

Hohaus, Stefan

Hokamp, Nils GroBe

Hołda, Mateusz Krystian

Höllig, Anke

Holobar, Aleš

Holzinger, Andreas

Horiguchi, Takashi

Hörmann, Gregor

Horne, Malcolm

Horsburgh, Ann

Horwath-Winter, Jutta

Hosohata, Keiko

Hostiuc, Sorin

Höti, Naseruddin 
Hou, Yanjun

Houde, Peter

Hough, Kenneth

Howard, Brittany E.

Hsiao, Kuei-Yang

Hsiao, Sheng-Mou

Hsiao, Yi-Hsuan

Hsieh, Ming-Ching

Hsieh, Tsung-Han

Hsieh, Tusty-Juan

Hsu, Bang-gee

Hsu, Chung-Yuan

Hsu, Chun-Nan

Hsu, Li-Yueh

Hsu, Yi-Chiung

$\mathrm{Hu}$, Baoli

$\mathrm{Hu}$, Taobo

$\mathrm{Hu}$, Ying

Huang, Chih-Chung

Huang, Huachao

Huang, Marilyn

Huang, Sarah Xuelian

Huang, Tzu-Ting

Huang, $\mathrm{Xu}$

Huang, Yao-Kuang

Hudlikar, Rasika

Hueso, Miguel

Hughes, Sean P. F.

Hund, Martin

Hung, Kuo-Chuan

Huss, Andre

Hwang, Eun Seong

Hwang, Jae Youn

Hwang, Sung Ho

Hwang, Yi-Ting

Iacopi, Elisabetta

Iacoviello, Massimo

Iasevoli, Felice

Ibrahim, Sherif Abdelaziz

Ide, Kazuki

Idicula, Titto T.

Ientile, Riccardo

Ieraci, Alessandro

Ignat, Anca

Ijare, Omkar B.

Ilardi, Federica

Iles, Ray

Ilhan, Harun

Ilias, Ioannis

Illert, Lena

Im, Hyung-Jun
Im, Hyungsoon

Imamura, Teruhiko

Imbimbo, Bruno

Inbar-Feigenberg, Michal

Indraccolo, Ugo

Inglada-Pérez, Lucía

Ingravallo, Giuseppe

Innaro, Nadia

Inomata, Minehiko

Inoue, Kazushi

Inoue, Takamitsu

Inusa, Baba

Inzani, Frediano

Iqbal, Hafiz M. N.

Iravani, Amir

Irie, Daisuke

Irisawa, Atsushi

Irzyniec, Tomasz

Isayama, Hiroyuki

Isaza Martínez, José Hipólito

Ishikawa, Tomohiro

Iskander, Robert

Isola, Gaetano

István, Hornyák

Itenov, Theis Skovsgaard

Ito, Takamichi

Itonaga, Masahiro

Iwata, Junichi

Iyer, Janaki

Izake, Emad L.

Izawa, Kazuhiro P.

Izonin, Ivan

Izumi, Gentaro

Izumi, Kouji

Jabłonowski, Zbigniew

Jabłońska, Beata

Jacob, Noam

Jafari, Jafar

Jagodzinski, Linda L.

Jahrami, Haitham

Jaidev Chakka, Leela Raghava

Jaime, Diego Franco

Jakobsen, Marianne A.

Jalan-Sakrikar, Nidhi

Jambor, Ivan

Janakiraman, Harinarayanan

Jang, Sung Ho

Jankauskiené, Augustina

Jans, Judith J. M.

Janzen, Nils

Jaouen, Vincent 
Jape, Gayatri

Jarosz-Chobot, Przemysława

Jårvinen, Tero A. H.

Jastrzebska, Agnieszka

Jeannot, Emilien

Jeerapan, Itthipon

Jenssen, Christian

Jeong, Hwal Rim

Jeong, Keun-Yeong

Jerebtsova, Marina

Jeremy, Teoh Yuen Chun

Jeruszka-Bielak, Marta

Jeschke, Udo

Ježek, Jan

Jezela-Stanek, A.

Jezierski, Tadeusz

Jiang, Chunjie

Jiang, Huailei

Jiang, Jianxiong

Jiang, Xuezhi

Jiao, Zhicheng

Jimenez Garcia, Victoria Alejandra

Jing, Ran

Jochelson, Maxine

Jochum, Christoph

Jødal, Lars

Johansson, Mats W.

Johns, Terrance G.

Johnson, Jason Michael

Jones, Mark

Jönsson, Daniel

Joyner, Chester J.

Ju, Wei-Jhong

Juhasz, Bela

Juhasz, Csaba

Jung Bae, Yun

Jung, Klaus

Jung, Sungmok

Junglee, Naushad Ali

Junker, Klaus

Kachlik, David

Kaden, Marika

Kaese, Sven

Kaewput, Wisit

Kagawa, Masaharu

Kagiyama, Nobuyuki

Kaigorodova, Evgeniya

Kaindl, Angela

Kajtár, Béla

Kakkassery, Vinodh

Kakoti, Ankana
Kalef-Ezra, John A.

Kalinin, Alexandr

Kallner, Anders

Kalogianni, Despina

Kalogirou, Charis

Kamata, Ken

Kaminski, Rafal

Kamolz, Lars P.

Kan, Chung-Dann

Kang, Jeonghyun

Kant, Sashi

Kanyong, Prosper

Kapischke, Matthias

Kapočiūtè-Dzikienè, Jurgita

Karayiannakis, Anastasios

Karbownik-Lewińska, Małgorzata

Karhula, Sakari

Karlinger, Kinga

Karlsson, Joakim

Karmazyn, Morris

Karoor, Vijaya

Karpiński, Tomasz M.

Karshikoff, Bianka

Karteris, Emmanouil

Karvouniaris, Marios

Karvounis, Artemios

Kashiwagi, Shinichiro

Kashtalap, Vasily

Kasprzak, Jaroslaw

Katagiri, Hiroshi

Kataoka, Masako

Katayama, Takayuki

Kate, Mahesh

Kato, Katsuhiko

Kaufmann, Walter E.

Kauppinen, Anu

Kaushik, Aniruddha

Kawabata, Hideaki

Kawanami, Daiji

Kawano, Kouichiro

Kawasaki, Hideya

Kazui, Toshinobu

Kelcz, Frederick

Keller, Brandis

Kellett, John G.

Kelley, Thomas

Kerman, Kagan

Kesmarky, Gabor

Kessler, Ronald C.

Khalek Abdel Razek, Ahmed Abdel

Khan, Amjad 
Khan, Krishnendu

Kharche, Sanjay

Khodanovich, Marina

Khokhar, Bilal

Kholmukhamedov, Andaleb

Kholová, Ivana

Khushi, Matloob

Kiesewetter, Dale O.

Kikuchi, Shogo

Kikuyama, Masataka

Kim, Beom Kyung

Kim, Bonglee

Kim, Dal Young

Kim, Hyungjoo

Kim, Hyun-Soo

Kim, Jee Taek

$\mathrm{Kim}$, Jin Su

Kim, Jin-woo

Kim, Jungyoon

Kim, Raymond H.

Kim, Ryong Nam

Kim, Seongho

Kim, Seongjae

Kim, Seong-Tae

Kim, Tae Hyun

Kim, Yerim

Kim, Yeun-Yoon

Kim, Yong Jin

Kimber-Trojnar, Żaneta

Kinfe, Thomas M.

Kinkorová, Judita

Kirac, Iva

Kisand, Kai

Kishaba, Tomoo

Kiss, Rita

Kistenev, Yurii

Kitrungrotsakul, Titinunt

Kittl, Sonja

Klapper, Stephen R.

Klar, Maximilian

Klatt, Dieter

Klebe, Sonja

Kleczynski, Pawel

Klobučar, Hrvoje

Klymenko, Yuliya

Knezevic, Jelena

Knudsen, Jonas R.

Koch, Achim

Koga, Fumitaka

Koivusalo, Antti Ilmari

Kojima, Takashi
Kolberg, Hans-Christian

Kolesar, Jill Marie

Kolonko, Aureliusz

Koltz, Michael T.

Komuraiah, Myakala

Kondru, Naveen

Kones, Richard

Kong, Kien Voon

Konishi, Masaaki

Konoike, Naho

Kontos, Christos K.

Koo, Kyo Chul

Koolivand, Abdollah

Kooyman, David L.

Kopechek, Jonathan

Koper-Lenkiewicz, Olga Martyna

Kopyta, Ilona

Koshiyama, Masafumi

Kosiak, Wojciech

Kosmala, Aleksander

Kosmas, Panos

Kost, Gerald

Kostkiewicz, Magdalena

Kostrzewa-Nowak, Dorota

Kosuke, Matsubara

Koszelewski, Dominik

Kotani, Toru

Kothapalli, Sri-Rajasekhar

Kotter, Elmar

Kotyla, Przemyslaw J.

Kourou, Konstantina

Kovács, Árpád

Kovács, Árpád Ferenc

Koyama, Eiki

Koyama, Takashi

Kraaijeveld, Adriaan O.

Kramvis, Anna

Krasiński, Zbigniew

Krawiec, Paulina

Krazinski, Bartlomiej Emil

Kreimer, Simion

Kreiser, Kornelia

Krewer, Carmen

Krick, Stefanie

Kriegova, Eva

Krishna, Somashekar G.

Kristensen, Gitte

Kroiss, Alexander

Krupa-Kozak, Urszula

Krzych, Lukasz

Krzyżak, Artur Tadeusz 
Krzyżanowski, Arkadiusz

Krzyżewski, Roger

Kshirsagar, Prakash G.

Kubera, Marta

Kubo, Eri

Kuboto, Kazuo

Kuddus, Ruhul H.

Kuessel, Lorenz

Kuhn, Elisabetta

Kulczyk, Tomasz

Kullaa, Arja

Kumar, Brajesh

Kumar, Gaurav

Kumar, Suneel

Kumaraswamy, Chitrala

Kumari, Sonam

Kummer, Kai

Kung, Hank F.

Kunicki, Michał

Kunz, Wolfram

Kuo, Ming-Tse

Kurasova, Olga

Kurata, Akira

Kuriakose, Maju

Kurir, Tina Tičinović

Kurzyna, Marcin

Kushima, Miki

Kushkevych, Ivan

Kusunose, Kenya

Kutikhin, Anton G.

Kutsumi, Hiromu

Kuwabara, Yoshihiro

Kuwahara, Takamichi

Kwak, HyoSung

Kwon, Seong Jung

Kyo, Satoru

La Forgia, Daniele

La Fougère, Christian

La Gatta, Annalisa

La Mura, Vincenzo

La Rosa, Valentina Lucia

La Vignera, Sandro

Labrou, Nikolaos

Labrune, Philippe

Lachmann, Robert

Lacina, Lukas

Laganà, Antonio Simone

Lahooti, Hooshang

Lai, Kent

Lai, Xin

Laihia, Jarmo
Lajolo, Carlo

Lam, Wai-Ching

Lam, Wilbur

Lamango, Nazarius S.

Lambrou, George I.

Lançon, Christophe

Landberg, Eva

Landegger, Lukas D.

Lang, Di

Lang, Hans Peter

Lang, Michael

Lang, Nikolaus Wilhelm

Lanza, Michele

Łapaj, Łukasz

Lapini, Alberto

Laptoiu, Dan

Larin, Kirill

Larsen, Anders Christian

Latosinska, Agnieszka

Lattanzi, Riccardo

Laura, Evangelista

Lavdaniti, Maria

Lazăr, Daniela Cornelia

Lazzarotto, Tiziana

Le Page, Cécile

Le, Thi-Thu-Huong

Lebogang, Lesedi

Lee, Alvin J. X.

Lee, Byung Kook

Lee, Changho

Lee, Chia-Hwa

Lee, Chii-Ming

Lee, Chu-Yu

Lee, Dong Yun

Lee, Heedoo

Lee, Hsiang-Chieh

Lee, Jeong Won

Lee, Jong Yun

Lee, Joo Hyoung

Lee, Juhyun

Lee, Kijoon

Lee, Ki-Sun

Lee, Kyung-Yil

Lee, Lukas Jyuhn-Hsiam

Lee, Maria

Lee, Matthew Meng Yang

Lee, Ming Jie

Lee, Philhyu

Lee, Posen

Lee, Regent

Lee, Sung Ki 
Lee, Tim

Lee, Tsung-Hsien

Lee, Wei-Chieh

Lee, Young-kyun

Lee, Young-Sil

Lee, Zhenghong

Leenen, Luke L. P. H.

Lei, Wei

Leibowitz-Amit, Raya

Lejniece, Sandra

Lemstra, Afina Willemina Evelien

Lenghel, Lavinia Manuela

Leoncini, Lorenzo

Leopoulou, Marianna

Leru, Poliana

Lesauskaite, Vaiva

Lesser, Thomas G.

Levine, Elliot M.

Levy, Steven

Lezot, Frederic

Li, Chenyu

Li, Chien-Feng

Li, Chunhui

Li, Dong

Li, Fuhai

Li, Kang

Li, Mei-Ling

Li, Meng

Li, Rong

Li, Wei-Ming

Li, Xiang

Li, Xiaopeng

Li, Xin

Li, Ying

Li, Yvonne

Liang, Jun

Liang, Yan

Liao, Wupeng

Liapis, George

Liau, Nicholas P. D.

Liberale, Gabriel

Lichtenauer, Michael

Lidbury, Brett

Lin, Chun-Li

Lin, Hsiang-Yu

Lin, Hsueh-chun

Lin, I-Cha

Lin, Tz-Feng

Linder, Ewert

Lindqvist, Johan

Linek, Paweł
Linhartová, Petra Bořilová

Lio, Do

Lithgow, Brian

Litofsky, Norman Scott

Little, Peter

Litvinova, Larisa

Litwin, Tomasz

Liu, GuanQun

Liu, Guanshu

Liu, Guigen

Liu, Jui-Ming

Liu, Minghua

Liu, Nana

Liu, Yu

Liu, Yueh-Wei

Liu, Zhixia

Livaniou, Evangelia

Llobet, Rafael

Lo Muzio, Lorenzo

Lobo, Joao

Lodi, Alessia

Loellgen, Herbert

Loffroy, Romaric

Loghin, Catalin

Loglio, Alessandro

Lojkić, Ivana

Lomonaco, Tommaso

Lonardo, Amedeo

Long, Meixiao

Longo, Dario Livio

Lopez-Escamez, Jose A.

López-Mora, Diego Alfonso

Lopez-Rincon, Alejandro

López-Urrutia, Eduardo

LoPresti, Patrizia

Lops, Diego

Lorenz Risch, Lorenz

Lorusso, Alessio

Losi, Maria Angela

Lu, Chia-Feng

Lu, Jing

Lu, Lingeng

$\mathrm{Lu}$, Shuiyu

Luangmonkong, Theerut

Lubas, Arkadiusz

Lubecka-Pietruszewska, Katarzyna

Lucarelli, Giuseppe

Lucchese, Alessandra

Ludmir, Ethan B.

Lukasiewicz, Aleksander

Lukoševičius, Arūnas 
Lunt, Peter

Luo, Ji-Dung

Luo, Xian

Luzzago, Stefano

M'kacher, Radhia

Ma, Suk Ling

Maas, Mario

Maboni, Grazieli

Maccio, Antonio

Macek, Pawel

Machairiotis, Nikolaos

Macq, Benoit

Madani, Afarine

Madea, Burkhard

Madeddu, Paolo

Madonov, Pavel

Madrigal, Irene

Maeda, Kazuhiro

Maffei, Angelo

Magan-Fernandez, Antonio

Magi-Galluzzi, Cristina

Magnaldo, Thierry

Mahan, J. Robert

Mahshid, Sara

Maiega, Hamidou

Mair, Johannes

Mais, Valerio

Majchrzak, Tomasz

Majewski, Sebastian

Majolino, Domenico

Majtner, Tomáš

Majumder, Mrinmoyee

Makavos, George

Makieva, Sofia

Makki, Malek

Makkiya, Mohammed

Maksemous, Neven

Malafoglia, Valentina

Malagelada, Francesc

Malaguarnera, Michele

Malapelle, Umberto

Malatino, Lorenzo

Malek, Lukasz

Malla, Spundana

Malli, Foteini

Mallidi, Srivalleesha

Mallineni, Sreekanth Kumar

Malouf, Camille

Manali, Effrosyni

Mancusi, Costantino

Mancuso, Salvatrice
Mandal, Subhamoy

Mandato, Claudia

Manenti, Antonio

Manfredini, Fabio

Mangino, Giorgio

Mango, Lucio

Mangogna, Alessandro

Manini, Paola

Mannelli, Lorenzo

Männistö, Tuija

Mansourian, Marjan

Mäntele, Werner

Mantripragada, Venkata P.

Marateb, Hamid Reza

Marc, Laine

Marchi, Antonella

Marcon, Magda

Marcu, Loredana

Mare, Ruxandra

Marella, Gian Luca

Margalit, Ili

Maria, Naomi I.

Mariano, Caratozzolo

Marinelli, Luca

Marino, Bruno

Mario, Pagnoni

Markić, Joško

Markiewicz, Aleksandra

Markman, Maurie

Markopoulos, Anastasios

Markopoulos, Georgios S.

Markou, Athina

Marks, Robert S.

Mármol, Ines

Marotte, Hubert

Marquering, Henk A.

Marques, João Gama

Marques, Tania

Marrelli, Massimo

Marshall, Andrew G.

Martaindale, Sarah

Marteau, Philippe R.

Martin, Diana L.

Martin, Maria

Martinelli, Fabio

Martinez-Camblor, Pablo

Martini, Katharina

Martins, Andre F.

Marto, Joana

Martucci, Gennaro

Marzano, Flaviana 
Masahiro, Sato

Masci, Lorenzo

Mascitti, Marco

Maspero, Cinzia

Mastoraki, Aikaterini

Mastropietro, Alfonso

Masumoto, Hidetoshi

Masuzaki, Ryota

Matczuk, Anna Karolina

Materazzi, Stefano

Mateus, Maria L.

Matheve, Thomas

Mathew, Ashish J.

Mathiessen, Alexander

Matozaki, Takashi

Matsubara, Edson Takashi

Matsuda, Atsushi

Matsumoto, Naoki

Matsuoka, Teruyuki

Matsusaki, Takashi

Matsushima, Takashi

Matsuzaki, Kentaro

Mattapallil, Joseph

Matynia, Anna

Maurea, Nicola

Maurea, Simone

Maw, Anna M.

Mayer, Philipp

Mayer, Rupert

Mazza, Elisa

Mbagwu, Smart Ikechukwu

McClure, Patrick

McDermott, John H.

McKay, Tina

Mcmorrow, Tara

McNeill, David Moreno

Mcsharry, Charles

Meder, Roger

Medipally, Dinesh

Mędrzycka, Wioletta

Meduri, Alessandro

Medvedev, Ilya Nikolaevich

Meehan, Katie

Mehariya, Sanjeet

Mehta, Noshir

Mehta, Sunali

Mehterov, Nikolay

Mei, Shenglin

Meignan, Michel

Meimandi, Kiana Jafari

Melaiu, Ombretta
Melas, Marilena

Meleady, Paula

Melling, Nathaniel

Melo, Bernardete F.

Menahem, Solomon

Mercadal, Lucile

Merkely, Béla

Merks, Hans

Mermis, Joel D.

Merolla, Francesco

Merrick, Daniel

Mesa, Francisco

Mesalam, Ahmed

Meshram, Nirvedh

Messineo, Ludovico

Mestrovic, Tomislav

Metaxas, George

Metovic, Jasna

Metzinger, Laurent

Meulstee, Jene W.

Meyer, Hans-Jonas

Mia, Sobuj

Miazek, Arkadiusz

Michael, Michael

Michálek, Pavel

Migita, Ohsuke

Mihaicuta, Stefan

Mikaïl, Nidaa

Miki, Kojiro

Miki, Yasuhiro

Mikropoulos, Christos

Mileti, Ilaria

Miller, Sara E.

Miller, Tamara P.

Minaga, Kosuke

Mincher, David

Minet, Olaf

Miotla, Pawel

Mirzakhani, Hooman

Miserocchi, Giuseppe

Mishra, Jay S.

Misiak, Błazej

Misiolek, Maciej

Mitchell, John S.

Mitchell, Ulrike H.

Mitrofanova, Alla

Mitrut, Radu

Miyata, Yasuyoshi

Miyazaki, Masaru

Miyoshi, Norio

Mizrahi, Joseph 
Mlynarska, Agnieszka

Mo, Lein-Ray

Mo, Makoto

Moadel, Renee M.

Moaven, Omeed

Moccia, Marcello

Modahl, Cassandra

Mohamed, Salah A.

Mohsin, Sadia

Molina-Alarcón, Milagros

Molina-Leyva, Alejandro

Moller, Ingrid

Møller, Søren

Moncayo, Roy

Monda, Emanuele

Monsalve, Maria

Monsuez, Jean-Jacques

Montagne, Axel

Monteiro, Luis

Monteiro, Sílvia

Moore, Matthew D.

Moore, Ronald B.

Moore, Stuart

Morales Narváez, Eden

Moran, Cesar A.

Moran, Oscar

Moretti, Matteo

Morga, Rafał

Mori, Kiyoshi

Morio, Yoshiteru

Mornos, Cristian

Moro, Alessandro

Morris, Daniel

Morris, Ulrika

Moscalu, Mihaela

Motakis, Efthymios

Mounien, Lourdes

Mozos, Ioana

Mrówczyńska, Maria

Mucci, Viviana

Mueller, Geoffrey

Muhammad, Khan

Mukaida, Naofumi

Müllauer, Leonhard

Mumoli, Nicola

Muniraj, Nethaji

Muniyan, Sakthivel

Muñoz-Berbel, Xavier

Munteanu, Florentina-Daniela

Murakami, Naoka

Murata, Soichiro
Muro, Shigeo

Murph, Mandi

Murphy, Caroline S.

Murphy, Ronan

Muscatiello, Nicola

Mußbacher, Marion

Muthupillai, Raja

Nabeta, Takeru

Nachmias, Boaz

Naeije, Robert

Nagai, Norihiro

Nagaoka, Masanori

Nagaraja, Haikady N.

Nagarajan, Prabakaran

Nagayasu, Eiji

Nagy, Magdolna

Naicker, Nisha

Nakachi, Tatsuya

Nakagami, Gojiro

Nakagawa, Naoki

Nakahara, Ichiro

Nakai, Yousuke

Nakajima, Takahito

Nakamura, Yoshiyuki

Nakanishi, Hiroyuki

Nakano, Toshiaki

Nakayama, Haruo

Nakayama, Hideaki

Nakayama, Jun

Nambiar, Dhanya K.

Nanetti, Lorenzo

Naňka, Ondřej

Naotake, Tsuda

Napoletano, Chiara

Napoli, Pietro

Narayan, Prakash

Nardella, Elizabeth

Nardini, Roberto

Nascimento, Gustavo G.

Nasierowska-Guttmejer, Anna

Natarajan, Sathish Kumar

Nath, Narayan Chandra Deb

Natsis, Konstantinos

Nault, Marie-Lyne

Navakauskienė, Rūta

Navarro, Estanis

Navran, Arash

Nayak, Nihar

Naydenov, Stefan

Nazzaro, Gianluca

Neginskaya, Maria A. 
Nemeș, Roxana Maria

Németh, Balázs

Nemeth, Norbert

Nenna, Antonio

Neogi, Ujjwal

Neri, Luca

Neri, Manuela

Nesbitt, Warwick S.

Nestrasil, Igor

Neubauer, Hans

Neuhaus, Ain A.

Nevler, Naomi

Nezhat, Farr Reza

Nguyen, Anh

Nguyen, Binh

Nguyen, Tin

Nguyen, Trieu

$\mathrm{Ni}$, Yicheng

Niaura, Gediminas

Nicolai, Eleonora

Niczyporyk, Jowita Samanta

Nie, Liming

Niedzialkowski, Pawel

Nii, Masafumi

Nikaki, Alexandra

Nikita, Efthymia

Nikolai, Bryan C.

Nikolaidis, Athanasios

Nikolaidis, Pantelis T.

Nikolic, Dejan

Nikolova, Irina

Nilghaz, Azadeh

Nilsson, Inga-Lena

Nilvebrant, Johan

Nioi, Matteo

Niraula, Boris B.

Nishida, Naoki

Nishii, Tatsuya

Nishio, Mizuho

Nishioka, Shinta

Niu, Tianhua

Nivedita, Nivedita

Nixon, Daniel W.

Nociari, Marcelo

Nogami, Makoto

Nohe, Anja

Nonnemacher, Michael R.

Noorani, Imran

Nopp, Anna

Norcic, Gregor

Nordman, Ina
Noríaki, Tomura

Nosch, Daniela Sonja

Noszczyk, Bartłomiej Henryk

Noventa, Marco

Novo-Veleiro, Ignacio

Novroski, Nicole

Novy, Jan

Nowicki, Michał

Nucera, Eleonora

Nucera, Riccardo

Nucifora, Gaetano

Nurwidya, Fariz

Nuvoli, Susanna

Nwabudike, Lawrence Chukwudi

Nylander, Karin

Nyman, Jeffry

O'Loughlin, Declan

Oakey, Zackery

Obermüller, Nicholas

Ochoa-Reparaz, Javier

Oh, Hoon Kyu

Oh, JungHwan

Ohgami, Robert S.

Ohko, Kentaro

Ohlemiller, Kevin K.

Öhrfelt, Annika

Okada, Hiroshi

Okajima, Hideaki

Okonogi, Noriyuki

Oldan, Jorge

Oleksiewicz, Urszula

Oliva, Francesco

Oliveira, Arlindo L.

Oliveira, Paula A.

Oliver, Eduardo

Olivera, Marco

Olivetti, Elena Carlotta

Olmedillas-López, Susana

Oltra, Elisa

Omar, Syed Haris

Omoumi, Patrick

Omura, Junichi

Ono, Yuko

Oriol, Albert

Orlacchio, Arturo

Orosz, Tamás

Ortega, Miguel Angel

Ortiz, Carmen

Orzechowski, Arkadiusz

Oshima, Yusuke

Osna, Natalia 
Osztheimer, István

Othman, Ahmed E.

Ottaiano, Alessandro

Ottenheijm, Ramon P. G.

Ouahabi, Abdeldjalil

Ovsepian, Saak V.

Owczarczyk-Saczonek, Agnieszka

Paal, Peter

Paço, Maria

Pafili, Kalliopi

Pagani, Marco

Pahk, Kisoo

Pal, Debjani

Palazón-Bru, Antonio

Palencia-Madrid, Leire

Palma, Marzia

Palmucci, Stefano

Palumbo, Carlotta

Palumbo, Giuseppe

Pampush, James

Pan, Luying

Panchapakesan, Balaji

Panda, Santosh

Pandit, Harshul

Panzuto, Francesco

Paone, Gaetano

Papageorgiou, Elpiniki

Papageorgiou, Ismini E.

Papalia, Rocco

Papamichail, Konstantinos

Papanas, Nikolaos

Papandrianos, Nikolaos

Papathanakos, Georgios

Papathanasiou, Maria

Paplińska-Goryca, Magdalena

Papp, John

Parenti, Ilaria

Parikesit, Arli Aditya

Parikh, Hemang

Parimon, Tanyalak

Paris, Debora

Park, Hoon

Park, Hyesun

Park, Jeongjin

Park, Kay-Hyun

Park, Min

Park, So Hyun

Parlatescu, Ioanina

Parnetti, Lucilla

Parravano, Mariacristina

Pasta, Gianluigi
Pasto, Anna

Pastores, Gregory M.

Pathak, Dhruba

Patowary, Ashok

Pavesio, Carlos

Pavlidis, Efstathios

Pavlik, Edward J.

Pavlov, Chavdar S.

Pavlova, Elena

Pavlu, Dagmar

Pawar, Shrikant D.

Pawlak, Katarzyna

Payabvash, Seyedmehdi Mehdi D.

Payne, Karl Frederick Braekkan

Peckham, Gabriel D.

Pecorelli, Anna

Pei, Yonggang

Peitsidis, Panagiotis

Pellacani, Giovanni

Pellegrini, Marco

Pellegrino, Gerardo

Pellicano, Rinaldo

Peng, Henry T.

Peng, Xianlu Laura

Perales, Alfredo

Peralta, Cleisson Fábio Andrioli

Peran, Ivana

Pereira, Bruno

Pereira, Filipe

Pereira, Maria A.

Pereira, Sofia

Pérez-García, Felipe

Pérez-Rodrigo, Carmen

Perillo, Letizia

Perisetti, Abhilash

Perlman, Sharon

Perls, Thomas T.

Pero, Raffaela

Peroz, Ingrid

Perri, Francesco

Perrucci, Gianluca Lorenzo

Perry, Anamarija M.

Pertinhez, Thelma

Pesce, Alessandro

Petejová, Nadežda

Petera, Jiri

Petersen, Lars J.

Petersen, Sven

Petersson, Fredrik

Petrosino, Maria

Petrov, Dmitry 
Pfeiffer, David C.

Piacenti, Ilaria

Piatek, Rafał J.

Piccin, Andrea

Piccinin, Elena

Piccoli, Martina

Piciu, Doina

Pienar, Corina

Pierucci, Paola

Pignatti, Patrizia

Pilli, Nageswara

Pilmane, Māra

Pincus, Matthew R.

Pinheiro, Joaquim M. B.

Pinho, Armando J.

Piorkowski, Adam

Piotrowska, Ewa

Piotrowski, Wojciech

Piovesan, Alessandro

Pirina, Pietro

Pirozzi, Christopher J.

Pisapia, Pasquale

Piskin, Senol

Pita, Sebastian

Pittner, Stefan

Piubelli, Chiara

Piva, Terrence

Pluschke, Gerd

Poddighe, Dimitri

Podgorski, Iva I.

Poenar, Daniel

Poetsch, Ansgar

Poirier, Yannick

Polańczyk, Andrzej

Poldermans, Don

Polewczyk, Anna

Polguj, Michal

Politano, Luisa

Polkowski, Wojciech

Pollari, Francesco

Polovina, Snezana P.

Poltorak, Lukasz

Pomara, Cristoforo

Pomari, Elena

Pond, Amber L.

Pontikos, Nikolas

Ponzetti, Marco

Ponzoni, Maurilio

Pop, Cristina

Pop, Tudor Lucian

Popescu, Mihaela R.
Poplawski, Tomasz

Popovic, Zoya

Poradowski, Dominik

Porpora, Maria Grazia

Porro, Chiara

Portelli, Marco

Pósa, Anikó

Pourlis, Aris

Powell, Folami Lamoke

Powrózek, Tomasz

Prakash, KN Bhanu

Prasad, Vikas

Pravdin, Sergei

Prchal, Josef T.

Pribilova, Anna

Principia, Scavo Maria

Printza, Nikoleta

Prodromidou, Anastasia

Protonotarios, Alexandros

Pruna, Dario

Przybylik-Mazurek, Elwira

Przybyło, Małgorzata

Pshezhetsky, Alexey

Psuj, Grzegorz

Pucci, Mairi

Pugliese, Nicola Riccardo

Puglisi, Fabio

Pugni, Lorenza

Pulavarti, Surya V. S. R. K.

Pulford, Karen

Pulito, Claudio

Puolakkainen, Mirja

Puri, Kriti

Puthenparampil, Marco

Puzzovivo, Agata

Qadura, Mohammad

Qasim, Muhammad

Qi, Qian-Rong

Qian, Xuejun

Qiu, Jiantai Timothy

Qiu, Zhen

Quattrone, Andrea

Que, Jenny

Queiro, Rubén

Quiróz-Mercado, Hugo

Rabkin, Simon W.

Rabuazzo, Agata Maria

Radzikowska, Elzbieta

Raeeszadeh-Sarmazdeh, Maryam

Rafi, Junaid

Ragavan, Mukundan 
Ragazzoni, Aldo

Rahelić, Dario

Rahman, Hafizur

Rahman, Masmudur M.

Rahman, Quazi

Rahman, Safikur

Raikhy, Gaurav

Raimondi, Lavinia

Raimondo, Diego

Rainieri, Enzo

Raizman, Rose

Rajda, Cecília

Raju, Nagarajan

Ramamurthy, Nitin

Ramasamy, Ranjan

Ramos, Carmen

Rampanelli, Elena

Ramprasath, Tharmarajan

Rana, Jyoti

Rapado-González, Óscar

Rapi, Stefano

Rapisarda, Agnese Maria Chiara

Rapone, Biagio

Räsänen, Esa

Rashid, Mohammad Harun

Rastogi, Madhup

Ratiu, Andreea

Rattray, Zahra

Raveglia, Federico

Raya, Ana

Raz, Eytan

Razvan-Cosmin, Petca

Rebelo, Sandra

Reddy, Sakamuri

Reddy, Sudhir Putty

Redente, Elizabeth F.

Rega, Daniela

Reichenbach, Zachary Wilmer

Ren, Jun

Ren, Ping

Rengo, Marco

Renz, Diane

Renzini, Alessandra

Requena, Manuel

Restaino, Stefano

Reyes-Aldasoro, Constantino Carlos

Rezania, Shahabaldin

Rezus, Ciprian

Ribaldone, Davide

Ribeiro, Ricardo

Ribera, Jordi
Ricardo, Riberio

Riccardo, Zoja

Ricchiuto, Piero

Richter, Wito

Riechelmann, Herbert

Riethmüller, Christoph

Riis, Margit L. H.

Riou, Laurent M.

Ripa, Rasmus Sejersten

Rizzello, Francesca

Rizzo, Cristiano

Rizzo, Stefania

Roberts, Drucilla J.

Roby, Justin A.

Rocchi, Anna

Rocco, Danilo

Roceanu, Adina

Rochani, Ankit

Rochtus, Anne M.

Ročka, Saulius

Rodrigo, Juan P.

Rodrigo-Muñoz, José M.

Rodrigues-de-Souza, Daiana Priscila

Rodríguez Hermosa, Juan Luis

Rodríguez, William R.

Rodríguez-Almagro, Daniel

Rodríguez-Sanz, David

Roedel, Franz

Rogobete, Alexandru Florin

Roje, Damir

Rojo, Maria Angeles

Rola, Radosław

Rolla, Giovanni

Romano, Lorenzo

Romero, Marta R.

Romero-Castro, Rafael

Ronen, Ohad

Rong, Chan Kuan

Rooney, Claire

Rosado, Carlos

Rosario, Barranco

Rosca, Monica

Rose, Christopher F.

Ross, Ryan

Rossi, Francesca

Rossi, Gemma

Rossi, Riccardo

Rossitto, Giacomo

Rossow, Lindy M.

Rostoker, Guy

Roubalova, Katerina 
Roumiguie, Mathieu

Rovas, Linas

Rovina, Davide

Rovnak, Joel

Rowley, K. Michael

Roy, Dhruvajyoti

Rozerta, Sokou

Rubegni, Pietro

Rubello, Domenico

Rudroff, Thorsten

Rundo, Leonardo

Ruscitti, Piero

Russell, Darren

Russo, Alessandro

Russo, Alessia

Russo, Giorgio

Rusy, Lynn M.

Rutkauskas, Saulius

Ružauskas, Modestas

Ruzzini, Laura

Rychtáriková, Renata

Ryozawa, Shomei

Ryswyk, Emer Van

Sabatino, Giovanni

Sabiniewicz, Robert

Saccardi, Carlo

Sachar, David B.

Sachithanandan, Nirupa

Sachpekidis, Christos

Saczonek, Agnieszka Owczarczyk

Sadahira, Takuya

Sadasivam, Mohanraj

Sahoo, Prativa

Sahoo, Subhransu

Sahrmann, Philipp

Saigusa, Daisuke

Saijo, Yoshifumi

Saini, Shikha

Sakai, Fumikazu

Sakamoto, Ryuichi

Sakamoto, Shinichi

Sakthivel, Kogularasu

Sakuma, Kunihiro

Salagierski, Maciej

Salehzadeh-Yazdi, Ali

Salerno, Sergio

Salido Tercero, Jesús

Salomone, Alberto

Salvador, Pablo

Salvi, Massimo

Sam, Ramin
Sánchez-Ávila, Ronald Mauricio

Sánchez-Bueno, Francisco

Sánchez-Gómez, Rubén

Sánchez-González, José-María

Sancisi, Valentina

Sanders, Matthew

Sandwall, Peter

Sanguedolce, Francesca

Santander, Petra

Santarelli, Andrea

Santhanam, Abirami

Santoro, Claudia

Santos, João H. P. M.

Santos, Rute

Santosh, K. C.

Santucci, Annalisa

Santulli, Gaetano

Sanz, Alejandro

Saravanakumar, Kandasamy

Sardu, Celestino

Sarkar, Amrita

Sarria-Santamera, Antonio

Sasaki, Eiji

Sasase, Tomohiko

Sato, Akira

Sato, Eiichi

Sato, Takashi Shawn P.

Savchenko, Andrey A.

Savoia, Paola

Sawicki, Marcin

Saynor, Zoe

Sazonova, Margarita A.

Sbrollini, Agnese

Scacco, Salvatore

Scarano, Antonio

Scaravilli, Vittorio

Scarlata, Simone

Scelsa, Barbara

Schäfer, Valentin S.

Schares, Gereon

Scharffenberg, Martin

Schell, Michael

Schepisi, Giuseppe

Schiattarella, Antonio

Schiavone, Marco

Schiff, Elena R.

Schiffer, Eric

Schildberg, Frank A.

Schillaco, Orazio

Schiller, Adalbert

Schirinzi, Tommaso 
Schmidl, Doreen

Schmitz, Ralf

Schmoll, Dieter

Schmutz, Axel

Schoenhagen, Paul

Schott, Ulf

Schreiber, Stefanie

Schrier, Rachel

Schröder, Agnes

Schuetz, Gunnar

Schulz, Wolfgang A.

Schwind, Sebastian

Scimeca, Manuel

Scisciola, Lucia

Seghaye, Marie-Christine

Sehgal, Chandra M.

Sen, Sougata

Senanayake, Sameera

Sensi, Matteo

Seong, Jin-Taek

Serafín, Verónica

Serafini, Gianluca

Seravalle, Gino

Serban, Daniela Elena

Sergi, Consolato

Sério, Susana

Serpico, Rosario

Serry, Mohamed

Sevvana, Madhumati

Seydel, Karl

Shackelford, Rodney E.

Shah, Jainil

Shah, Neeraj

Shahzad, Atif

Shamshirband, Shahab

Shan, Hongming

Shankar, Eswar

Shao, Wei

Shapiro, Adrienne

Sharara, Fady

Sharker, Shazid Md.

Sharma, Gaurav

Sharma, Nisha

Sharon, Denise

Shaw, Alyra J.

Shaw, George J.

Sheikh, Alaullah

Shen, Liming

Shen, Ming-Yuan

Shen, Shichen

Sheu, Jim
Shi, Junchao

Shi, Lei

Shibukawa, Goro

Shih, David

Shih, Ie-Ming

Shiina, Marisa

Shimada, Yohta

Shimatani, Masaaki

Shimizu, Yuji

Shinde, Aparna

Shintaku, Haruo

Shirai, Jyunsuke

Shirshin, Evgeny

Shivakumar, Pranav

Shoji, Sunao

Shokoohi, Hamid

Shukla, Kirtikar

Siddiqui, Imran

Sidorov, Denis Nikolai

Sidorov, Evgeny

Sier, Cornelis

Signore, Alberto

Siiskonen, Teemu

Sijens, Paul E.

Sikalidis, Angelos

Sikora, Mariusz

Silva, Carlos

Silva, Catarina

Silva, Marcelo Sousa

Silva, Susana Nunes

Simakov, Sergey

Simka, Marian

Simko, Fedor

Simmonds, Rachel

Simoes, Joana

Simon, Thomas

Simonov, Nikolai

Simopoulou, Mara

Šindlerová, Lenka

Singh, Amrita

Singh, Kamayani

Singh, Pankaj K.

Singh, Pratibha

Singh, Sandeep

Singh, Sukhvinder

Singh, Sumit Randhir

Singhal, Tarun

Sinha, Niharika

Sinning, Christoph

Sireci, Federico

Sisti, Giovanni 


\author{
Sjödahl, Gottfrid \\ Skoczyński, Szymon \\ Škrlec, Ivana \\ Sleigh, James \\ Slezák, Radovan \\ Sliwinska-Mosson, Mariola \\ Sloan, Kenneth \\ Śmigiel, Robert \\ Smith, David F. \\ Smith, Gary A. \\ Smith, Heather F. \\ Smith, Lloyd M. \\ Smyczyńska, Joanna \\ Snyder, Christopher S. \\ Sobhani, Navid \\ Sobolewski, Eric \\ Socea, Bogdan \\ Sochaj-Gregorczyk, Alicja Maria \\ Sohns, Jan Martin Sommerlath \\ Soica, Codruta \\ Solé Cañadas, Carla \\ Solé, Cristina \\ Soler, Carles \\ Solimando, Antonio Giovanni \\ Solís-Lemus, José Alonso \\ Solitro, Giovanni \\ Somboonwit, Charurut \\ Sommariva, Elena \\ Song, Chang Myeon \\ Song, In-Seok \\ Sorrentino, Francesco Saverio \\ Sorsa, Timo \\ Sotnikov, Dmitriy V. \\ Spagnolo, Elvira Ventura \\ Spalice, Alberto \\ Spang, Christoph \\ Spano, Giuseppe \\ Spanu, Angela \\ Spartalis, Eleftherios \\ Speranza, Domenico \\ Sperti, Cosimo \\ Spong, Catherine \\ Spooner, Neil \\ Sporea, Ioan \\ Sprinkart, Alois M. \\ Sreedharan, Sreejesh \\ Sridhar, Subbaramiah \\ Staboulidou, Ismini \\ Stacey, Michael C. \\ Štajduhar, Ivan \\ Stanciu, Gabriela-Dumitrita
}

Stanculeanu, Dana Lucia

Standley, Claire J.

Stanley, Jone A.

Starshinova, Anna Andreevna

Stawski, Robert

Stecco, Alessandro

Stefanidis, Constantinos J.

Stefanini, Lucia

Stefano, Alessandro

Stefanovic, Ivan

Stefanowicz, Joanna

Steibliene, Vesta

Steinbichler, Teresa Bernadette

Steponaitis, Giedrius

Stepp, Mary Ann

Steyger, Peter

Stiglic, Gregor

Stillo, Francesco

Stoffman, Jayson

Stoian, Dana

Stonbraker, Samantha

Strati, Areti

Straudi, Sofia

Strekalova, Tatyana

Struck, Manuel

Sturiale, Carmelo Lucio

Stutz, Bernardo

Su, Chih-Min

Su, Kuo-Chih

Subedi, Dinesh

Subhi, Yousif

Suciu, Bogdan Andrei

Suga, Jennifer

Sugiura, Tsuyoshi

Suhail, Yasir

Sujit, Sheeba Jenifer

Sukocheva, Olga

Sulikowski, Piotr

Sumi, Yasuo

Sun, Daekyu

Sun, Li

Sun, Zhixiong

Sun, Zhonghua

Sung, Wen-Wei

Surani, Salim

Suraweera, Amila

Sutoh, Yoichi

Suzuki, Motofumi

Suzuki, Shugo

Suzuki, Yoriyasu

Swami, Umang 
Syafrudin, Muhammad

Syeda-Mahmood, Tanveer Fathima

Szabó, Dóra

Szczepanek, Joanna

Szlubowski, Artur

Szoboszlai, Norbert

Szopa, Andrzej

Szubert, Sebastian

Szutkowski, Kosma

Taffé, Patrick

Tagliabracci, Adriano

Tajika, Tsuyoshi

Tajiri, Naoki

Takabatake, Kiyofumi

Takabayashi, Shuji

Takač, Iztok

Takado, Yuhei

Takaki, Manabu

Takamatsu, Shigeyuki

Takebayashi, Shigeo

Taked, Takayuki

Takeda, Hiroyuki

Takedatsu, Hidetoshi

Takeishi, Naoki

Takeuchi, Rikiya

Talaat, Iman M.

Tallmadge, Rebecca

Tamaki, Nobuharu

Tan, Karen

Tan, Ning

Tan, Wenbin

Tanaka, Naoro

Tanaka, Takashi

Tang, Fangfang

Tangaro, Sonia

Tanner, Nathan

Tanyeri, Melikhan

Tao, Jeremiah P.

Taradaj, Jakub

Tarantino, Giovanni

Tashima, Toshihiko

Tavares, João Manuel R. S.

Tayek, John A.

Taylor, Andy

Taylor, Matthew

Teague, Shawn D.

Tedaldi, Gianluca

Teixidó, Cristina

Teng, Ru-Jeng

Teragawa, Hiroki

Terayama, Hayato
Terracciano, Daniela

Tesarik, Jan

Teteloshvili, Nato

Teus, Miguel

Tha, Khin Khin

Thakur, Sunitha N.

Than, Nandor Gabor

Thekkinkattil, Dinesh K.

Thevenot, Paul T.

Thior, Ibou

Thirunavukkarasu, Shyamala

Thomsen, Thomas

Thomssen, Christoph

Thornell, Ian M.

Tian, Furong

Tian, Weihua

Timchenko, Pavel

Timchenko, Tatiana

Timmons, Mark K.

Tinelli, Andrea

Tito, Raúl Yhossef

Tjioe, Marco

Tjong, Sie Chin

Tkáč, Ján

Todsen, Tobias

Toelle, Brett

Toews, Matthew

Togawa, Yaei

Tokarek, Tomasz

Tokas, Theodoros

Tolmachev, Vladimir

Tomatsu, Shunji

Tomczyk, Arkadiusz

Tomescu, Mirela Cleopatra

Tomsone, Signe

Tonet, Elisabetta

Tong, Yat-Ching

Tonni, Gabriele

Toriello, Filippo

Török, Peter

Torras-Ambròs, Joan

Torres, Jorge

Torres-Sangiao, Eva

Toshchakov, Stepan

Tosi, Davide

Tosun, Duygu

Toth, Kalman

Touil-Boukoffa, Chafia

Tovoli, Francesco

Toyos, Melissa

Tozzo, Pamela 
Trainor, Patrick

Trecroci, Athos

Trendelenburg, Marten

Treviño, Mercedes

Trimarchi, Matteo

Trimboli, Pierpaolo

Trinci, Margherita

Tripodo, Claudio

Triunfo, Stefania

Trouillas, Jacqueline

Trubiani, Oriana

Trudel, Dominique

Tsai, James

Tsai, Kun-Lin

Tsai, Rongkung

Tsakanikas, Panagiotis

Tsang, Kwong Yok

Tsangaris, Iraklis

Tsoi, James Kit-hon

Tsubota, Akihito

Tsuchiya, Junichi

Tsuchiya, Takayoshi

Tsunematsu, Takaaki

Tu, Hung-Pin

Tuovinen, Tero

Turiel, Maurizio

Turner, Jonathan

Turner, Richard

Tutino, Vincent M.

Tuttolomondo, Antonino

Twarock, Sören

Tyan, Yu-Chang

Tyler, Nichole

Tzortzakakis, Antonios

Tzschatzsch, Heiko

Tzur, Tamar

Uchiyama, Koji

Ueda, Koji

Ugga, Lorenzo

Ugon, Adrien

Ulivi, Paola

Ullah, Amin

Ullah, Irfan

Umano, Giuseppina Rosaria

Umapathy, Ganesh

Umemori, Juzoh

Umemura, Atsushi

Umutlu, Lale

Unchwaniwala, Nuruddin

Ungurianu, Anca

Upadhyaya, Jasbir
Urbano, Nicoletta

Urbanska, Edyta Maria

Uribe, Alberto A.

Usai Satta, Paolo

Usui, Noriyoshi

Utsunomiya, Daisuke

Uyar, Denise S.

Vaculovičová, Marketa

Vafaee, Manouchehr

Vaibhav, Kumar

Vaira, Valentina

Valentinčič, Nataša Vidovič

Valenzuela-fernández, Agustín

Valverde, Jose R.

Van Bussel, Bas C. T.

Van Den Berge, Margreet

Van Den Meiracker, Anton H.

Van Der Burgt, Ineke

Van Etten, Ellis S.

Van Hal, Sebastiaan

Van Heerden, Peter Vernon

Van Rhee, Frits

Van Timmeren, Janita

Van Trijffel, Emiel

Vancheri, Federico

Vandenbriele, Christophe

Vanzi, Francesco

Vardhanabhuti, Varut

Varela, Gonzalo

Varela-Nieto, Isabel

Vasefi, Maryam

Vashist, Sandeep Kumar

Vaskelyte, Jolanta-Justina

Vassiliou, Aliki Georgia

Vaughan, Christopher L.

Vaverka, Miroslav

Vaz, Pedro

Vecchio, Michele

Veenland, Jifke

Vegas-Sánchez-Ferrero, Gonzalo

Veiga, Maria Isabel

Veilleux, Louis-Nicolas

Velan, Sendhil

Veldeman, Michael

Velikova, Tsvetelina

Venkataraman, Rajesh

Venkatesh, Alagarswamy G.

Venkateswaran, Mahima

Ventura, Paolo

Venturella, Roberta

Versari, Annibale 
Vidili, Gianpaolo

Vieira, Edgar Ramos

Viggiano, Andrea

Viggiano, Davide

Vilahur, Gemma

Villani, Rosanna

Vincent, Amy E.

Vincenzi, Colombina

Virella, Daniel

Virumbrales-Muñoz, María

Visentin, Silvia

Vitale, Giuseppe

Vitale, Salvatore Giovanni

Vitoratou, Silia

Vitorino, Rui Miguel Pinheiro

Vlamos, Panayiotis

Vlk, Martin

Vo, Dat

Voellger, Benjamin

Vogel, Wouter V.

Voiculescu, Dan

Volaklis, Konstantinos

Volkau, Ihar

Von Arx, Claudia

Von Blanckenburg, Pia

Vora, Mehul

Vos, Cornelis

Vrachatis, Dimitrios A.

Vriens, Dennis

Vujović, Igor

Wagner, Kay-Dietrich

Wakabayashi, Hiroshi

Walocha, Jerzy

Wan, Shibiao

Wang, Bin

Wang, Ceming

Wang, Chen-Yu

Wang, Chun-Hou

Wang, Dongwen

Wang, Dongyi

Wang, Hsiang-Chen

Wang, Hsiuying

Wang, Huanyu

Wang, Kai

Wang, Maosen

Wang, Mengyu

Wang, Shao-Hung

Wang, Tsung-Jen

Wang, William

Wang, Xue

Wang, Yeng-Tseng
Wang, Yi

Wang, Yixiang

Wang, Yumeng

Wang, Zhanxiang

Wang, Zhaoning

Wansbrough-Jones, Mark

Ward, Mark

Warsof, Steven L.

Warzecha, Zygmunt

Washington, Anthony Valance

Watabe, Tadashi

Waterhouse, Miguel

Watier, Bruno

Watson, Des

Wawruszak, Anna

Weaver, Kathryn Nicole

Webster, Bonnie

Webster, Dianne

Weglarz, Wladyslaw

Wegrzyn, Grzegorz

Wehrhahn, Michael

Wei, Wei-Chyou

Weiner, Juliane

Wells, Jack

Wełnicki, Marcin

Werner, Erica

Westhoff, Niklas

Wetz, Christoph

Whiteley, Mark S.

Widera, Marek

Widerska-Chadaj, Zaneta S.

Wiechowska-Kozłowska, Anna

Wiehe, Arno

Wielgos, Miroslaw

Wietrzyk, Joanna

Wilczek, Piotr

Wilkinson, Nafisa

Williams, E. Mark

Wilson, Alphus D.

Winn, Bryan

Witczak, Carol A.

Witowski, Jan

Wojsyk - Banaszak, Irena

Wolfram, Frank

Wong, Kwong-Kwok

Wong, Maurice

Wong, Season S.

Wong, Yon-Cheong

Woo, Sungmin

Worrell, Stephanie

Woźniak, Mateusz 


\begin{tabular}{|c|c|}
\hline Wozniak-Knopp, Gordana & Yeh, Hong-Zen \\
\hline Wróblewska-Seniuk, Katarzyna & Yeh, Randy \\
\hline Wrona, Ewa & Yeo, Sujung \\
\hline Wu, (Jason) Boyang & Yerlikaya, Gülen \\
\hline Wu, Chau-Chung & Yin, Changchuan \\
\hline Wu, Chiao-En & Yin, Meng \\
\hline Wu, Chueh-Hung & Yip, Bon Ham \\
\hline $\mathrm{Wu}$, Chun-Hu & Yokoi, Fumiaki \\
\hline Wu, Hsien-Tsai & Yokokawa, Tetsuro \\
\hline Wu, Wei-Ting & Yokoyama, Yujiro \\
\hline Wujtewicz, Magdalena & Yoneda, Takashi \\
\hline Wysocki, Marian & Yong, Kar Wey \\
\hline Xiang, Handan & Yoo, Jae-Sung \\
\hline Xiao, Perry & Yoo, Jung Sun \\
\hline Xie, Jiexiong & Yordanov, Angel \\
\hline Xie, Lingxi & Yoshida, Hiroshi \\
\hline Xie, Wankun & Yoshida, Yoshio \\
\hline Xie, Yibin & Yu, Jian Q. \\
\hline Xu, Chenchu & Yu, Jinxing \\
\hline Xu, Guan & Yu, Ollie Yiru \\
\hline Xu, Guofang & Yu, Shaode \\
\hline Xu, Jinbin & Yu, Yanping \\
\hline Xu, Peng & Yu, Zeyun \\
\hline Xu, Rong & Yuan, Qi \\
\hline Xu, Wen Tao & Yuan, Zhihong \\
\hline Xu, Xiaowen & Yue, John K. \\
\hline Yadav, Dhananjay & Z'Graggen, Werner \\
\hline Yagmur, Eray & Zabotti, Alen \\
\hline Yahyaoui, Raquel & Zafar, Haroon \\
\hline Yamada, Hiroyuki & Zafrakas, Menelaos \\
\hline Yamada, Shuhei & Zagidullin, Naufal \\
\hline Yamada, Yukio & Zak, Marek \\
\hline Yamaguchi, Satoshi & Zakrocka, Izabela \\
\hline Yamasaki, Tricia & Zamarchi, Rita \\
\hline Yamauchi, Akira & Zamboni, Paolo \\
\hline Yan, Ke & Zamorano-Leon, José Javier \\
\hline Yan, Shengmin & Zamyatnin, Andrey \\
\hline Yan, Xuefeng & Zanetti, Elisabetta \\
\hline Yang, Chih-Jen & Zanin, Massimiliano \\
\hline Yang, Chih-Yu & Zannoni, Gian Franco \\
\hline Yang, Chun Zhang & Zaric, Svetislav \\
\hline Yang, Hao & Zauderer, Marjorie G. \\
\hline Yang, Joshua Y. C. & Zavattaro, Elisa \\
\hline Yang, Kuen-Cheh & Zavos, Christos \\
\hline Yang, Ling Li & Zeleznik, Oana Alina \\
\hline Yang, Peixin & Zelger, Bernhard \\
\hline Yang, Yeongwook & Zemouri, Ryad \\
\hline Yang, Zhihong & Zenarruzabeitia, Olatz \\
\hline Yao, Chaoqun & Zeng, Xiangyan \\
\hline Yaroslavsky, Anna N. & Zewdie, Ephrem Takele \\
\hline Yayan, Josef & Zhang, Chengzhi \\
\hline
\end{tabular}




\author{
Zhang, Chi \\ Zhang, Ershuai \\ Zhang, Hua \\ Zhang, Ming \\ Zhang, Qian \\ Zhang, Xinxing \\ Zhang, Xiujuan \\ Zhang, Yanfei \\ Zhang, Zhao \\ Zhang, Zhiguo \\ Zhao, Ningning \\ Zhao, Qian \\ Zhao, Yue \\ Zheng, Dandan \\ Zheng, Minzhang \\ Zhou, Qingyu
}

Zhou, Tianhao

Zhou, Zhuhuang

Zhurakivska, Khrystyna

Zibis, Aristeidis H.

Zicari, Alessandra

Zieger, Martin

Zieliński, Maciej

Ziemssen, Focke

Zimran, Ari

Zingarelli, Basilia

Ziółkowski, Robert

Zoli, Angelo

Zolotukhin, Igor A.

Zong, Weiwei

Zubrzycki, Jarosław

Zucchetta, Pietro 